\title{
Complete Response to Trastuzumab- Based Chemotherapy in a Patient with Human Epidermal Growth Factor Receptor-2-Positive Metastatic Salivary Duct Carcinoma ex Pleomorphic Adenoma
}

\author{
Shigenori Kadowaki ${ }^{a}$ Yasushi Yatabe $^{b}$ Hitoshi Hirakawa $^{c}$ \\ Azusa Komori $^{a}$ Chihiro Kondoh $^{a}$ Yasuhisa Hasegawa $^{c}$ Kei Muro ${ }^{a}$ \\ Departments of ${ }^{\mathrm{a}} \mathrm{Clinical}$ Oncology, ${ }^{\mathrm{b}} \mathrm{Pathology}$ and Molecular Diagnostics, and ${ }^{\mathrm{C}} \mathrm{Head}$ and \\ Neck Surgery, Aichi Cancer Center Hospital, Nagoya, Japan
}

\section{Key Words}

Trastuzumab · Carcinoma ex pleomorphic adenoma Salivary duct carcinoma $\cdot$ Epidermal growth factor receptor- 2 . Complete response

\begin{abstract}
Introduction: Carcinoma ex pleomorphic adenoma (CXPA) of the salivary glands has often a salivary duct carcinoma (SDC) component, which resembles ductal carcinoma of the breast and frequently overexpresses human epidermal growth factor receptor-2 (HER2). We report a case of metastatic CXPA with SDC component who was treated with trastuzumab-based chemotherapy and has had a durable complete response. Case Report: A 74-year-old man was diagnosed with CXPA of the right parotid gland. The resected tumor was histologically diagnosed as CXPA with a predominant SDC component that showed strong positivity for HER2 protein and HER2 gene amplification. Multiple pulmonary metastatic lesions were detected after surgery, and combination chemotherapy with paclitaxel and trastuzumab was initiated. A complete response was confirmed after 7 treatment cycles, and no evidence of disease progression has been observed after 13 months of initiation of therapy. Conclusions: This report suggests a potential utility of trastuzumab-based chemotherapy for HER2positive CXPA.




\section{Introduction}

Carcinoma ex pleomorphic adenoma (CXPA) is an aggressive salivary gland malignancy that predominantly affects the parotid gland and accounts for $11.6 \%$ of all salivary gland malignancies [1]. CXPA has two components: a typically high-grade epithelial malignancy that develops in association with a primary or recurrent benign mixed tumor. According to Lewis et al. [2], salivary duct carcinoma (SDC) is the second most frequent histological type of the malignant component (34\%), following adenocarcinoma not otherwise specified (44\%). SDC is a clinically aggressive neoplasm that bears a striking histological resemblance to ductal carcinoma of the breast. The majority of SDCs develop de novo; however, SDCs may develop from the malignant transformation of a pre-existing pleomorphic adenoma in 20$27 \%$ of cases [3-5]. The prognosis of SDC is poor; locoregional recurrences and distant metastases are frequently observed, resulting in a high mortality rate $[3,5,6]$.

Currently, there is no standard treatment for CXPA, although several phase II studies and case reports have explored the role of targeted agents in chemotherapy for advanced salivary gland cancers. Moreover, previous reports did not distinguish between CXPA and other histological subtypes, which further diminishes the available data regarding systemic chemotherapy for this subtype [7].

Immunohistochemical analyses show that human epidermal growth factor receptor-2 (HER2) is overexpressed in two thirds (21-92\%) of SDCs [6, 8-10]. This is also true in the case of CXPA with a SDC component; in a report by Hashimoto et al. [11], 85.7\% of such tumors were HER2 positive, whereas only $29.4 \%$ of cases of CXPA with other components were HER2 positive. Therefore, HER2 may be a potential therapeutic target in the SDC type CXPA; however, there is only anecdotal evidence for a clinical response of salivary gland cancer to trastuzumab-based chemotherapy [12-16].

Here, we report a case of CXPA that was predominantly SDC type and that became metastatic 5 months after surgery. The patient achieved complete response (CR) with paclitaxel and trastuzumab combination chemotherapy presumably because of the high level of HER2 gene amplification in the tumor. Because SDC is a common component of CXPA, trastuzumab treatment should be considered when the tumor is positive for HER2.

\section{Case Report}

A 74-year-old man noticed a hard, painless mass in his right parotid gland 2 months before his initial visit to our institution. His past medical history included prostate cancer, which was treated with definitive radiotherapy 2 years and 8 months ago. There was no evidence of facial nerve paralysis. Fine-needle aspiration biopsy findings of the parotid mass were consistent with those of CXPA. Positron emission tomography (PET)-computed tomography (CT) revealed an enhanced mass in the right parotid gland, without enlarged cervical lymph nodes. A total right parotidectomy along with right neck dissection and facial nerve reconstruction using cervical nerves was performed. Macroscopically, the tumor measured $25 \times 17 \mathrm{~mm}$ at its largest dimensions and had a cartilage-like whitish cut surface with focal invasion of the surrounding fat tissue (fig. 1a). The mass consisted of broad hyaline tissue with nests of tumor cells (fig. 1b). At least two tissue components were identified: high-grade carcinoma with a cribriform growth pattern and comedo-like necrosis in the nests and slit-like tubular epithelium with myoepithelial cells at the base (fig. 1c). CXPA with a predominant SDC component was diagnosed on the basis of the presence of a cartilaginous, hyaline, nodular background and benign pleomorphic adenoma in the nodule. 
CT performed 4 months after a radical surgery revealed recurrence in the right cervical nodes, which was treated by right neck dissection. The recurrent tumor was histologically diagnosed as SDC (fig. 1d). The primary tumor was positive (score 3+) for membranelocalized HER2/neu protein (Dako, Herceptest ${ }^{\mathrm{TM}}$ ) (fig. 2a). Dual-color chromogenic in situ hybridization revealed that HER2 gene was highly amplified (Ventana, INFORM HER2 Dual ISH) (fig. 2b). Five months later, CT revealed multiple bilateral pulmonary metastases (fig. $3 a)$, and systemic treatment with paclitaxel $\left(175 \mathrm{mg} / \mathrm{m}^{2}\right)$ and trastuzumab $(8 \mathrm{mg} / \mathrm{kg}$ dose loading followed by $6 \mathrm{mg} / \mathrm{kg}$ every 3 weeks) was initiated every 21 days. All pulmonary metastatic lesions had regressed after 4 treatment cycles (fig. 3b), and CR was confirmed after 7 cycles. The patient was maintained on trastuzumab alone $(6 \mathrm{mg} / \mathrm{kg}$ every 3 weeks), and no evidence of disease progression was observed at the last contact with the patient, which was approximately 13 months after initiation of therapy.

\section{Discussion}

CXPA shows a multistep progression from intraductal to invasive cancer. The increasing frequency of HER2 gene amplification increases with CXPA progression, suggesting that HER2 may play a key role in the progression of this tumor [17]. HER2, which encodes the $185-\mathrm{kDa}$ transmembrane glycoprotein with tyrosine kinase activity, is considered to play an important role in controlling cell growth and development. Amplification of HER2 and overexpression of HER2 protein are associated with poor prognosis in breast cancer. Trastuzumab binds to the extracellular domain of HER2 protein, inhibiting the proliferation of HER2-positive tumor cells. In clinical trials of HER2-positive metastatic breast cancer, trastuzumab has shown significant efficacy with regard to tumor response, resulting in improved survival [18].

Trastuzumab is now also being used to treat salivary gland cancers, with mixed results. In a phase II study of HER2-overexpressing metastatic salivary gland cancers, the overall response to trastuzumab monotherapy was only 7\% (1/14); only 1 patient with mucoepidermoid carcinoma attained partial response, and 2 patients with SDC had stable disease for 24 and 42 weeks, respectively [19]. This study was terminated early because of the low frequency of HER2 positivity and lower response than expected. In contrast, recently reported case reports of salivary gland cancers with HER2 overexpression have demonstrated the potential utility of trastuzumab-based chemotherapy [12-16]. In a report by Nabili et al. [12], CR was achieved in 1 of 3 patients with metastatic SDC in which HER2 was amplified and overexpressed. After trastuzumab therapy, lung metastases completely disappeared; this patient has remained disease free for 3 years. Similar case reports by Kaidar-Person et al. [13] and Prat et al. [14] confirmed CR after chemotherapy with trastuzumab, carboplatin, and paclitaxel in patients with HER2-positive SDC. The patients described by Kaidar-Person et al. [13] experienced CR of all pulmonary metastatic lesions 3 months after initial therapy. Prat et al. [14] reported CR of metastatic lesions in the lung, mediastinum, and liver after 3 treatment cycles. Nashed and Casasola [15] report a durable and complete disappearance of lung and liver metastases in response to docetaxel and trastuzumab combination therapy in a patient with HER2-positive SDC arising in pleomorphic adenoma. Sharon et al. [16] also report a dramatic response in a patient with HER2-overexpressing CXPA; after 3 cycles of capecitabine and trastuzumab, fluorine-18-fluorodeoxyglucose PET revealed no abnormal uptake in multiple bony metastases for more than 2 years.

Our patient's tumor was strongly positive for HER2 immunostaining and showed highlevel HER2 amplification; CR was achieved after initial treatment with paclitaxel and 
trastuzumab. Combination chemotherapy for HER2-positive SDC was a reasonable strategy because paclitaxel is more effective when used in combination with trastuzumab in HER2positive breast cancer [18]. Our result suggests that trastuzumab may be effective in highly specific subtypes of CXPA but should be interpreted with caution because this is a case report of a single patient. However, it is not practically feasible to conduct a prospective clinical trial using only this particular histological subtype; thus, case reports such as ours are of clinical significance. Taken together with the other case reports, these results indicate that HER2 status should be examined, particularly in SDC or CXPA with a SDC component. Further studies to evaluate the utility of trastuzumab-based therapy in HER2-positive SDC are warranted.

\section{Disclosure Statement}

The authors declare that they have no potential conflicts of interest.

\section{References}

1 Gnepp DR: Malignant mixed tumors of the salivary glands: a review. Pathol Annu 1993;28:279-328.

2 Lewis JE, Olsen KD, Sebo TJ: Carcinoma ex pleomorphic adenoma: pathologic analysis of 73 cases. Hum Pathol 2001;32:596-604.

3 Lewis JE, McKinney BC, Weiland LH, Ferreiro JA, Olsen KD: Salivary duct carcinoma. Clinicopathologic and immunohistochemical review of 26 cases. Cancer 1996;77:223-230.

4 Delgado R, Vuitch F, Albores-Saavedra J: Salivary duct carcinoma. Cancer 1993;72:1503-1512.

5 Hosal AS, Fan C, Barnes L, Myers EN: Salivary duct carcinoma. Otolaryngol Head Neck Surg 2003;129:720725.

-6 Jaehne M, Roeser K, Jaekel T, Schepers JD, Albert N, Loning T: Clinical and immunohistologic typing of salivary duct carcinoma: a report of 50 cases. Cancer 2005;103:2526-2533.

7 Surakanti SG, Agulnik M: Salivary gland malignancies: the role for chemotherapy and molecular targeted agents. Semin Oncol 2008;35:309-319.

-8 Skalova A, Starek, Kucerova V, Szepe P, Plank L: Salivary duct carcinoma - a highly aggressive salivary gland tumor with HER-2/neu oncoprotein overexpression. Pathol Res Pract 2001;197:621-626.

-9 Dagrada GP, Negri T, Tamborini E, Pierotti MA, Pilotti S: Expression of HER-2/neu gene and protein in salivary duct carcinomas of parotid gland as revealed by fluorescence in-situ hybridization and immunohistochemistry. Histopathology 2004;44:301-302.

10 Locati LD, Perrone F, Losa M, Mela M, Casieri P, Orsenigo M, Cortelazzi B, Negri T, Tamborini E, Quattrone P, Bossi P, Rinaldi G, Bergamini C, Calderone RG, Liberatoscioli C, Licitra L: Treatment relevant target immunophenotyping of 139 salivary gland carcinomas (SGCs). Oral Oncol 2009;45:986-990.

-11 Hashimoto K, Yamamoto H, Shiratsuchi H, Nakashima T, Tamiya S, Higaki Y, Komune S, Tsuneyoshi M, Oda Y: S100p expression in ductal type of carcinoma ex pleomorphic adenoma. Am J Surg Pathol 2011;35:346355.

12 Nabili V, Tan JW, Bhuta S, Sercarz JA, Head CS: Salivary duct carcinoma: a clinical and histologic review with implications for trastuzumab therapy. Head Neck 2007;29:907-912.

$\$ 13$ Kaidar-Person 0, Billan S, Kuten A: Targeted therapy with trastuzumab for advanced salivary ductal carcinoma: case report and literature review. Med Oncol 2012;29:704-706.

$\checkmark 14$ Prat A, Parera M, Reyes V, Peralta S, Cedres S, Andreu J, Huguet P, del Campo JM: Successful treatment of pulmonary metastatic salivary ductal carcinoma with trastuzumab-based therapy. Head Neck 2008;30:680683.

15 Nashed M, Casasola RJ: Biological therapy of salivary duct carcinoma. J Laryngol Otol 2009;123:250-252.

16 Sharon E, Kelly RJ, Szabo E: Sustained response of carcinoma ex pleomorphic adenoma treated with trastuzumab and capecitabine. Head Neck Oncol 2010;2:12.

-17 Hashimoto K, Yamamoto H, Shiratsuchi H, Nakashima T, Tamiya S, Nishiyama K, Higaki Y, Komune S, Tsuneyoshi M, Oda Y: HER-2/neu gene amplification in carcinoma ex pleomorphic adenoma in relation to progression and prognosis: a chromogenic in-situ hybridization study. Histopathology 2012;60:E131-E142.

18 Slamon DJ, Leyland-Jones B, Shak S, Fuchs H, Paton V, Bajamonde A, Fleming T, Eiermann W, Wolter J, Pegram M, Baselga J, Norton L: Use of chemotherapy plus a monoclonal antibody against HER2 for metastatic breast cancer that overexpresses HER2. N Engl J Med 2001;344:783-792. 


\section{Case Reports in Oncology}

\begin{tabular}{l|l}
\hline Case Rep Oncol 2013;6:450-455 \\
\hline DOI: 10.1159/000355219 & $\begin{array}{l}\text { @ 2013 S. Karger AG, Basel } \\
\text { www.karger.com/cro }\end{array}$ \\
\hline
\end{tabular}

Kadowaki et al.: Complete Response to Trastuzumab-Based Chemotherapy in a Patien with Human Epidermal Growth Factor Receptor-2-Positive Metastatic Salivary Duct Carcinoma ex Pleomorphic Adenoma

19 Haddad R, Colevas AD, Krane JF, Cooper D, Glisson B, Amrein PC, Weeks L, Costello R, Posner M: Herceptin in patients with advanced or metastatic salivary gland carcinomas. A phase II study. Oral Oncol 2003;39:724-727.
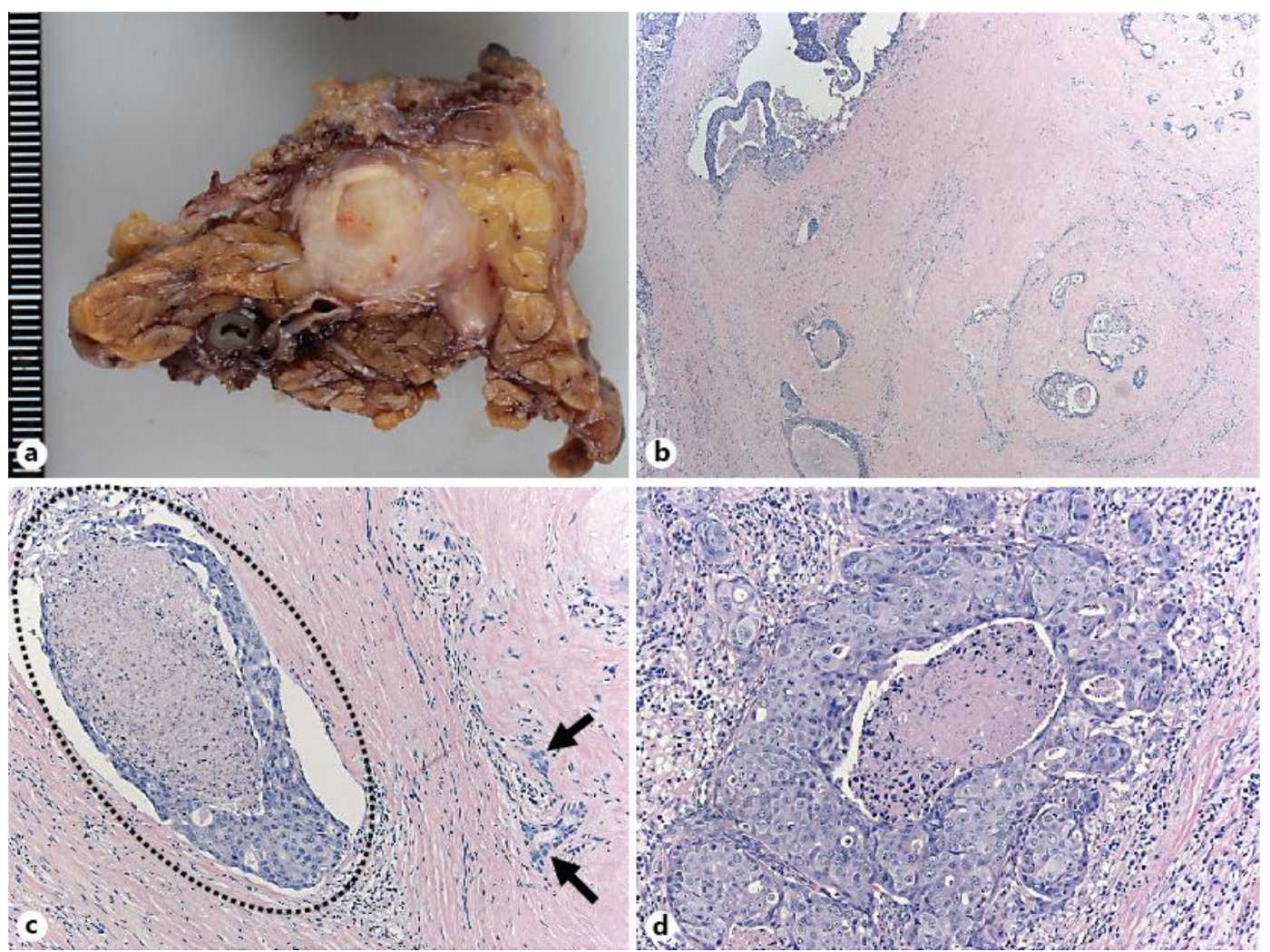

Fig. 1. a Macroscopic appearance of the parotid tumor resected. $\mathbf{b}$ In the low-power view, the nodule was comprised of hyaline tissue with nests of tumor cells. c As cellular contents, high-grade carcinoma with a cribriform growth pattern and comedo-like necrosis (dotted line) and slit-like tubular epithelium with myoepithelium (arrows) were identified. $\mathbf{d}$ Histological diagnosis of resected lymph nodes was SDC with comedo-like necrosis. 


\section{Case Reports in Oncology}

Case Rep Oncol 2013;6:450-455

DOI: $10.1159 / 000355219$

Kadowaki et al.: Complete Response to Trastuzumab-Based Chemotherapy in a Patien with Human Epidermal Growth Factor Receptor-2-Positive Metastatic Salivary Duct Carcinoma ex Pleomorphic Adenoma
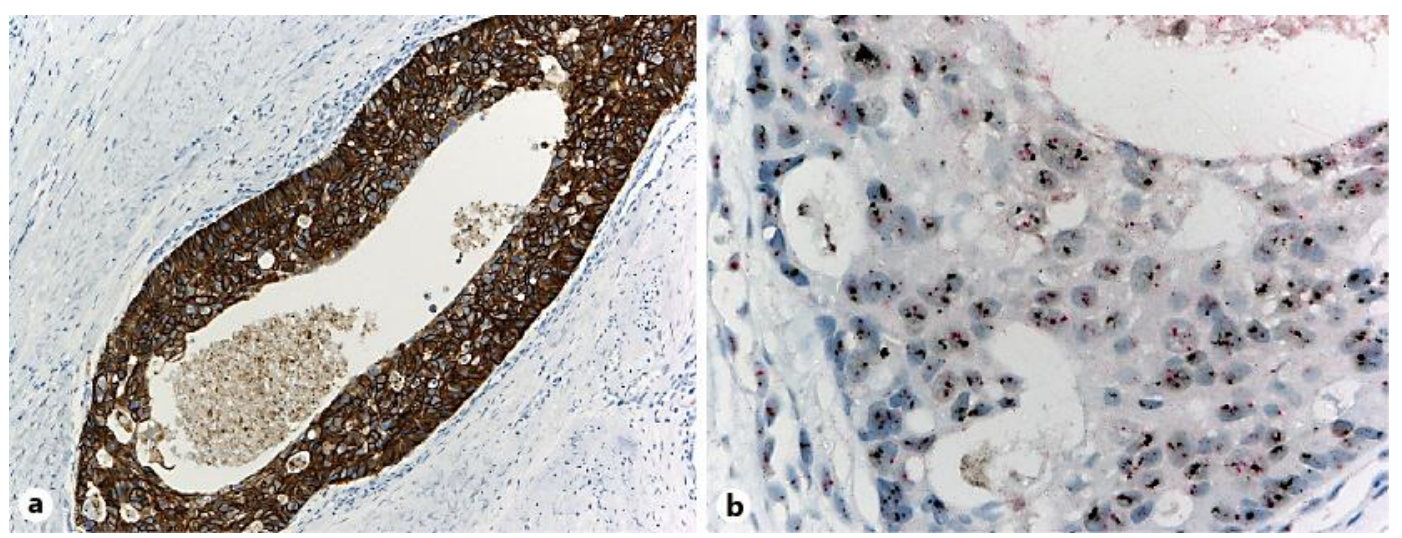

Fig. 2. a Immunohistochemical analysis revealed that the tumor cells were positive for HER2 overexpression (Dako, Herceptest). b Dual-color chromogenic in situ hybridization revealed that HER2 gene was highly amplified, with HER2:chromosome 17 centromere ratio at 10:1 or more (Ventana, INFORM HER2 Dual ISH). Red = Chromosome 17 centromere; black = HER2.

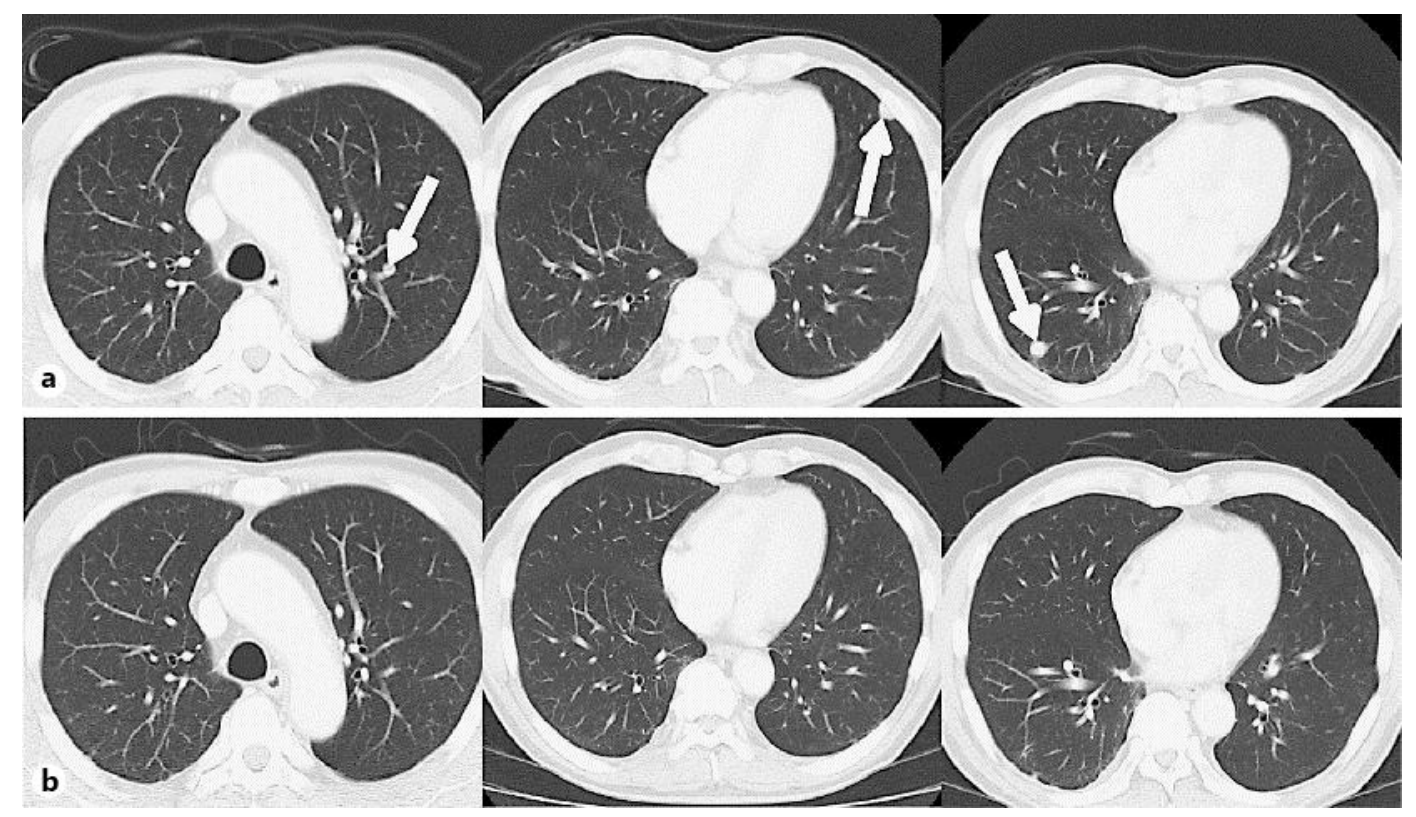

Fig. 3. a A thoracic CT scan revealed multiple bilateral pulmonary metastases (arrows). b After 4 cycles of chemotherapy with paclitaxel and trastuzumab, a CR of all pulmonary metastatic lesions was observed. 